\title{
Modified Hermite Pulse-Based Wideband Communication for High-Speed Data Transfer in Wireless Sensor Applications
}

\author{
Kushal P. Pradhan, Yang-Guo Li, A. K. M. Arifuzzman and Mohammad Rafiqul Haider * \\ Department of Electrical and Computer Engineering, University of Alabama at Birmingham (UAB), \\ Birmingham, AL 35294, USA; kpradhan@uab.edu (K.P.P.); yangguo@uab.edu (Y.-G.L.); arif@uab.edu (A.K.M.A.) \\ * Correspondence: mrhaider@uab.edu; Tel.: +1-205-975-0321
}

Received: 2 October 2017; Accepted: 28 November 2017; Published: 1 December 2017

\begin{abstract}
With technological advances in the field of communication, the need for reliable high-speed data transfer is increasing. The deployment of large number of wireless sensors for remote monitoring and control and streaming of high definition video, voice and image data, etc. are imposing a challenge to the existing network bandwidth allocation for reliable communication. Two novel schemes for ultra-wide band (UWB) communication technology have been proposed in this paper with the key objective of intensifying the data rate by taking advantage of the orthogonal properties of the modified Hermite pulse (MHP). In the first scheme, a composite pulse is transmitted and in the second scheme, a sequence of multi-order orthogonal pulses is transmitted in the place of a single UWB pulse. The MHP pulses exhibit a mutually orthogonal property between different ordered pulses and due to this property, simultaneous transmission is achieved without collision in the UWB system, resulting in an increase in transmission capacity or improved bit error rate. The proposed schemes for enhanced data rate will offer high volume data monitoring, assessment, and control of wireless devices without overburdening the network bandwidth and pave the way for new platforms for future high-speed wireless sensor applications.
\end{abstract}

Keywords: UWB; modified hermite pulse; orthogonal pulse; correlation; bit error rate

\section{Introduction}

In recent times, ultra-wide band (UWB) communication technology has become widely used in wireless sensing applications such as structural health monitoring, patient health care monitoring, military surveillance, and smart homes, etc. Owing to its attractive features like lower power consumption, its power spectrum below the noise floor, and its consistency with preexisting wireless technologies, there is a shift of interest among researchers towards the pulse-based UWB communication since narrow-band communication for WSN is afflicted with channel impairments and bandwidth constraints. In a UWB system, the narrow-band signal is a major interferer; however, a significant number of articles have been reported to mitigate these problems [1-5].

A lot of work has been put in towards developing innovative techniques for pulse set generation and modulation for UWB systems. Pulse-position amplitude modulation (PPAM) [6], biorthogonal-pulse position modulation (BPPM) [7], and OOK-PSM [8] are proposed in several works in the literature to achieve better system performance using combined modulation techniques with higher data rates, less complex receivers, and lower power consumption. The Federal Communications Commission (FCC) defines the regulation of pulse power and spectrum [9]. Different types of pulse shapes are used to obtain desired power and spectral ranges $[10,11]$.

One of the main drawbacks of the UWB system is the generation of proper orthogonal pulses and their higher derivatives. A modified Hermite pulse (MHP) set generator has been presented 
in [12] which uses an efficient technique to generate orthogonal pulses. Similarly, the authors of $[13,14]$ endorsed the practice of employing orthogonal pulse to increase the data rate in the prevailing UWB communication systems. The authors of [14] used different pulse in different positions to increase the number of information bits to be transmitted, whereas the authors of [13] utilized an orthogonal pulse sequence to encode the information bits. The orthogonal pulses can be added in time and transmitted as a composite pulse to the receiver end. These orthogonal pulses are then put through a correlator which in turn decodes the composition of the symbols.

UWB systems offer many advantages over conventional radio communications systems, e.g., low power consumption for transmission and much improved robustness to multipath interference [15]; as a result UWB technology is very suitable for indoor wireless channels that are very prone to multipath interference. Owing to the short time duration of its pulses, multipath components can be differentiated with ease while higher data rates can also be achieved. Different kinds of modulation schemes have already been proposed for obtaining improved data rates, which in general make use of pulse-position modulation (PPM) in addition to the employment of time-hopping sequences. The constraints associated with the Gaussian monopulse have posed many impediments in the generation of new pulses [16-20]. It has also been observed that the modified Hermite polynomials can be used to generate the pulses that can be implemented in an M-ary modulation scheme [21], which exploits the mutual orthogonality of such polynomials.

In this paper a novel M-ary pulse shape modulation (PSM) scheme for UWB communication technology has been proposed in order to enhance the data rate by exploiting the orthogonal properties of the MHP and increase the number of simultaneous data transmissions. The proposed work shows our previously reported orthogonal modified Hermite polynomial (MHP) pulse-based high data density wireless communication. Unlike existing schemes for orthogonal pulse generation, the developed mathematical model offers fewer required functional blocks, reduced system complexity, and enhanced power efficiency. In this work, instead of using a single orthogonal pulse, a series of pulses or a pulse sequence is used to represent each channel or user. All the pulse sequences from all the channels are then summed up with their respective time slots to create a unified pulse sequence. It is noteworthy that all the pulse sequences are not necessarily orthogonal to each other; however, due to the orthogonality property of the multi-order MHP pulses, as the pulse sequences are added up with their respective time slots, the individual pulse sequence is not affected or destroyed. With the proposed scheme, in a single-channel UWB system, the data rate has been improved by $n$-folds for $n$-number of MHP orthogonal pulses in a single time slot. For instance 16 distinct composite pulses can be represented through the employment of 4 MHP orthogonal pulses, resulting in a data rate enhancement of 4 folds in a single channel. On the other hand, for $n$-number of MHP orthogonal pulses the channel number has been increased to $n(n-1)$. Hence, a unique pattern sequence has been developed by encoding the MHP orthogonal pulses to transmit binary high bits for each channel. By using 4 orthogonal pulses the proposed scheme can generate distinctive pattern sequences for 12 channels.

The organization of this paper is as follows. Section 2 briefly describes the process of composite pulse generation while Sections 3 and 4 describe the single-user and multiple-user UWB systems, respectively. In Section 5, the bit error rate (BER) analysis has been illustrated. Finally in Section 6, the paper is summarized.

\section{Composite Pulse Generation}

Traditional Hermite polynomials have been defined by the authors of [22]. For the purpose of generating orthogonal pulses for UWB communications, MHPs have been proposed by pioneering researchers [23,24]; which permit one particular dynamic system to generate another MHP. Since orthogonal pulses can be transmitted simultaneously without mutual interference, orthogonal pulse set-based IR-UWB systems have the potential to achieve a higher data rate than the conventional IR-UWB systems [25-54]. Most of the mathematical functions for the design of orthogonal UWB pulses 
such as the Haar function [55], the modified Hermite polynomial (MHP) function [22-24,56], and the prolate spheroidal wave function (PSWF) [57,58], etc. involve complicated math models and power hungry hardware implementation [59]. MHP pulses, which have lower computational complexity than PSWF-based pulses, possess the properties of orthogonality and nearly-constant pulse widths irrespective of the pulse orders. However, the conventional MHP generation scheme as proposed in $[23,24]$ involves the second-order derivative and the squared time function, $t^{2}$, making the pulse generator complicated in computation. In our previously reported article [12], an innovative choice is made by following the coupled first-order dynamics to break down the second-order system as a combination of two first-order systems and sub-GHz operation to reduce the system complexity and power consumption of the orthogonal pulse generation. Owing to the simplified model, there are only 2 integrators, 5 multipliers, 3 adders, and a ramp signal generator in the system which is significantly less complex and power-efficient than the previous works [22].

An efficient mathematical model for MHP set generation has been presented in [12], where the $n$th order derivatives of the MHP are given as

$$
\begin{gathered}
\tau \dot{h}_{n}(t)=-\frac{t}{2 \tau} h_{n}(t)+n h_{n-1}(t) \\
\tau \dot{h}_{n-1}(t)=\frac{t}{2 \tau} h_{n-1}(t)-h_{n}(t)
\end{gathered}
$$

The proposed neuromorphic MHP set generator has been implemented in Cadence by virtue of Verilog-AMS. Figure 1 presents the zero- $\left(M H P_{0}\right)$, first- $\left(M H P_{1}\right)$, second- $\left(M H P_{2}\right)$ and third- $\left(\mathrm{MHP}_{3}\right)$ order modified Hermite pulses respectively, generated using the pulse set generator proposed in [12]. The MHP pulse is called the Gaussian mono-pulse. For higher order pulses the generator generates two complimentary pulses of the same order, which is evident in Figure 1.

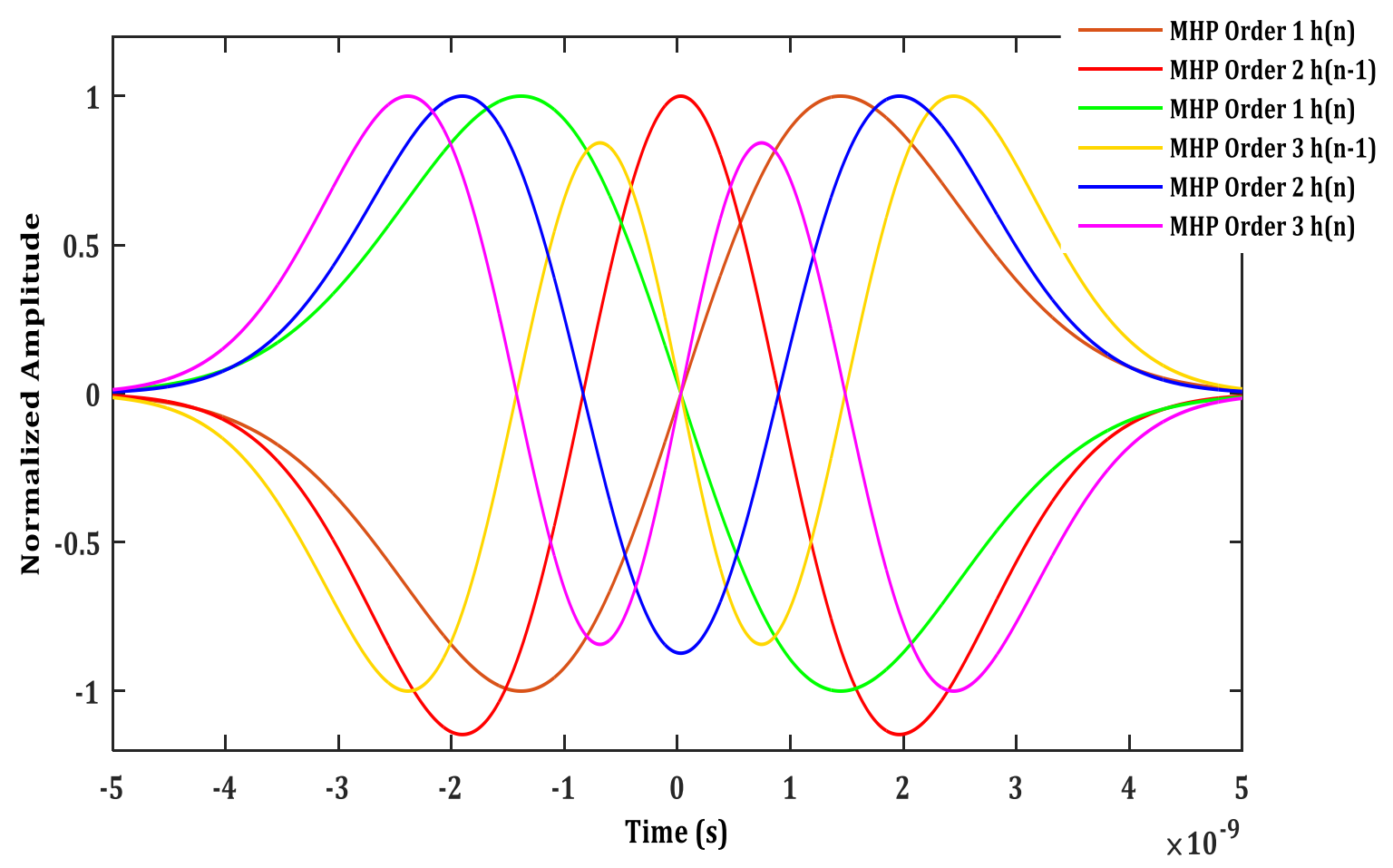

Figure 1. Combined plots of modified Hermite polynomial (MHP) order 0, 1, 2 and 3 from the generator circuit (pulse width $=10 \mathrm{~ns}$ ).

The distinguishing features of the orthogonal pulses being added up in the time domain and the successful recovery of the composition of the composite pulses make it possible to both boost the data 
rate and increase the number of channels for simultaneous transmission. Instead of transmitting the individual orthogonal pulses and modulating in the pulse polarity, position, and phase, this paper proposes the transmission of the composite pulse and modulation of the information in pulse shape. With the proposed scheme of data modulation for $n$ number of orthogonal pulses, the data rate in the single-user scenario was increased by $n$ folds and in the case of the multi-user scenario, it supported $n(n-1)$ channels for simultaneous data transmission.

Correlation analysis was performed using MATLAB in order to check the orthogonality of the generated pulses. Tables 1-3 present the correlation values between the orthogonal signals with pulse width of $6 \mathrm{~ns}, 10 \mathrm{~ns}$, and $18 \mathrm{~ns}$, respectively. The correlation value ranges from -1 to 1 , where 1 implies that there is a perfect correlation between the signals, 0 implies that there is no correlation between the signals and -1 implies that the signals are just opposite in nature. $h(n)$ and $h(n-1)$ are the orthogonal pulses generated with opposite phase and the addition of such complementary pulses, for instance $M H P_{0}=h(n)$, and $M H P_{0}=h(n-1)$, will cancel out each other. Hence it is vital that only one is chosen from the pair of generated complementary orthogonal pulses.For the proposed UWB system, $M H P_{0}=h(n), M H P_{1}=h(n), M H P_{2}=h(n-1)$, and $M H P_{3}=h(n)$ have been selected for their performance in providing better correlation results. Here, $M H P_{0}=h(n)$ and $M H P_{2}=h(n-1)$ are out of phase with $M H P_{2}=h(n)$ and $M H P_{3}=h(n)$.

Table 1. Correlation coefficient between the orthogonal pulses (6 ns).

\begin{tabular}{|c|c|c|c|c|c|c|c|}
\hline & $\begin{array}{c}M H P_{0} \\
h(n)\end{array}$ & $\begin{array}{c}M H P_{1} \\
h(n)\end{array}$ & $\begin{array}{c}M H P_{1} \\
h(n-1)\end{array}$ & $\begin{array}{c}M H P_{2} \\
h(n)\end{array}$ & $\begin{array}{c}M H P_{2} \\
h(n-1)\end{array}$ & $\begin{array}{c}\mathrm{MHP}_{3} \\
h(n)\end{array}$ & $\begin{array}{c}M H P_{3} \\
h(n-1)\end{array}$ \\
\hline$M H P_{0} h(n)$ & 1 & 0 & 0 & 0.3409 & -0.3208 & 0 & 0 \\
\hline$M H P_{1} h(n)$ & 0 & 1 & -1 & 0 & 0 & 0.1743 & -0.1913 \\
\hline$M H P_{1} h(n-1)$ & 0 & -1 & 1 & 0 & 0 & -0.1363 & 0.1538 \\
\hline$M H P_{2} h(n)$ & 0.3409 & 0 & 0 & 1 & -1 & 0 & 0 \\
\hline$M H P_{2} h(n-1)$ & -0.3208 & 0 & 0 & -1 & 1 & 0 & 0 \\
\hline$M H P_{3} h(n)$ & 0 & 0.1743 & -0.1363 & 0 & 0 & 1 & -1 \\
\hline $\mathrm{MHP}_{3} h(n-1)$ & 0 & -0.1913 & 0.1538 & 0 & 0 & -1 & 1 \\
\hline
\end{tabular}

Table 2. Correlation coefficient between the orthogonal pulses (10 ns).

\begin{tabular}{|c|c|c|c|c|c|c|c|}
\hline & $\begin{array}{c}M H P_{0} \\
h(n)\end{array}$ & $\begin{array}{c}M H P_{1} \\
h(n)\end{array}$ & $\begin{array}{c}M H P_{1} \\
h(n-1)\end{array}$ & $\begin{array}{c}M H P_{2} \\
h(n)\end{array}$ & $\begin{array}{c}M H P_{2} \\
h(n-1)\end{array}$ & $\begin{array}{c}M H P_{3} \\
h(n)\end{array}$ & $\begin{array}{c}M H P_{3} \\
h(n-1)\end{array}$ \\
\hline$M H P_{0} h(n)$ & 1 & 0 & 0 & 0.2507 & -0.2507 & 0 & 0 \\
\hline$M H P_{1} h(n)$ & 0 & 1 & -1 & 0 & 0 & 0.2507 & -0.2507 \\
\hline$M H P_{1} h(n-1)$ & 0 & -1 & 1 & 0 & 0 & -0.2507 & 0.2507 \\
\hline$M H P_{2} h(n)$ & 0.2507 & 0 & 0 & 1 & -1 & 0 & 0 \\
\hline$M H P_{2} h(n-1)$ & -0.2507 & 0 & 0 & -1 & 1 & 0 & 0 \\
\hline$M H P_{3} h(n)$ & 0 & 0.2507 & -0.2507 & 0 & 0 & 1 & -1 \\
\hline$M H P_{3} h(n-1)$ & 0 & -0.2507 & 0.2507 & 0 & 0 & -1 & 1 \\
\hline
\end{tabular}

Table 3. Correlation coefficient between the orthogonal pulses (18 ns).

\begin{tabular}{|c|c|c|c|c|c|c|c|}
\hline & $\begin{array}{c}M H P_{0} \\
h(n)\end{array}$ & $\begin{array}{c}M H P_{1} \\
h(n)\end{array}$ & $\begin{array}{c}M H P_{1} \\
h(n-1)\end{array}$ & $\begin{array}{c}M H P_{2} \\
h(n)\end{array}$ & $\begin{array}{c}M H P_{2} \\
h(n-1)\end{array}$ & $\begin{array}{c}M H P_{3} \\
h(n)\end{array}$ & $\begin{array}{c}M H P_{3} \\
h(n-1)\end{array}$ \\
\hline$M H P_{0} h(n)$ & 1 & 0 & 0 & 0.2541 & -0.2541 & 0 & 0 \\
\hline$M H P_{1} h(n)$ & 0 & 1 & -1 & 0 & 0 & 0.1968 & -0.1968 \\
\hline$M H P_{1} h(n-1)$ & 0 & -1 & 1 & 0 & 0 & -0.1968 & 0.1968 \\
\hline$M H P_{2} h(n)$ & 0.2541 & 0 & 0 & 1 & -1 & 0 & 0 \\
\hline$M H P_{2} h(n-1)$ & -0.2541 & 0 & 0 & -1 & 1 & 0 & 0 \\
\hline$M H P_{3} h(n)$ & 0 & 0.1968 & -0.1968 & 0 & 0 & 1 & -1 \\
\hline $\mathrm{MHP}_{3} h(n-1)$ & 0 & -0.1968 & 0.1968 & 0 & 0 & -1 & 1 \\
\hline
\end{tabular}


The correlation values between the selected orthogonal pulses and all possible combinations of 2, 3, and 4 orthogonal pulses are shown in Tables 4 and 5. The correlation coefficient values show how closely the two signals under consideration resemble each other. Hence, it can be interpreted as a conviction factor for the given MHP to be present in the composite pulse under inspection. For instance, for $57 \%$ confidence it can occur that $M H P_{0}$ is present in the composite pulse of $M H P_{0,1}$ (Table 4: second row, second column). From Tables 4 and 5, the maximum correlation value for the false positive is $16.22 \%$ (Table 4: third row, fourth column) for the correlation between $M H P_{1}$ and the combinations of $\mathrm{MHP}_{0,3}$. On the other hand, the minimum value for the true positive correlation is $24.42 \%$ (Table 5: second row, sixth column) for the correlation between $M H P_{0}$ and the combinations of $M H P_{0,1,2,3}$. This means that the correlation will give a maximum of $16.22 \%$ confidence in the worst case even if the pulse is not present in the composite pulse. Similarly, the correlation will give a minimum value of $24.24 \%$ confidence in the worst case even when the pulse is present in the composite pulse. Hence, the threshold value to determine if an orthogonal pulse is present or not in the given composite pulse is set to $21 \%$. This threshold value gives nearly $4 \%$ margin on both false positive and true positive correlation values. Any correlation coefficient value less than $21 \%$ would interpret that the signal is not present in the composite pulse. On the other hand, any correlation value greater than or equal to $21 \%$ will interpret that the orthogonal signal is present in the given composite pulse.

Table 4. Correlation coefficient values for selected pulses and all possible combinations of 2 orthogonal pulses.

\begin{tabular}{ccccccc}
\hline & $\mathbf{M H P}_{\mathbf{0 , 1}}$ & $\boldsymbol{M H P}_{\mathbf{0 , 2}}$ & $\mathbf{M H P}_{\mathbf{0 , 3}}$ & $\boldsymbol{M H P}_{\mathbf{1 , 2}}$ & $\mathbf{M H P}_{\mathbf{1 , 3}}$ & $\boldsymbol{M H P}_{\mathbf{2 , 3}}$ \\
\hline$M H P_{0}$ & 0.5698 & 0.4454 & 0.5664 & -0.1744 & 0 & -0.1736 \\
$M H P_{1}$ & 0.8218 & 0 & 0.1622 & 0.7275 & 0.7713 & 0.1438 \\
$M H P_{2}$ & -0.1448 & 0.7528 & -0.1440 & 0.6861 & 0 & 0.6829 \\
$M H P_{3}$ & 0.1617 & 0 & 0.8241 & 0.1432 & 0.7759 & 0.7305 \\
\hline
\end{tabular}

Table 5. Correlation coefficient values for selected pulses and all possible combinations of 3 and 4 orthogonal pulses.

\begin{tabular}{|c|c|c|c|c|c|}
\hline & $M H P_{1,2,3}$ & $M H P_{0,2,3}$ & $M H P_{0,1,3}$ & $M H P_{0,1,2}$ & $M H P_{0,1,2,3}$ \\
\hline$M H P_{0}$ & 0.1319 & 0.3165 & 0.4075 & 0.3178 & 0.2442 \\
\hline$M H P_{1}$ & 0.6594 & 0.1385 & 0.7043 & 0.7005 & 0.6450 \\
\hline$M H P_{2}$ & 0.5188 & 0.5349 & -0.1036 & 0.5372 & 0.4127 \\
\hline $\mathrm{MHP}_{3}$ & 0.6633 & 0.7037 & 0.7085 & 0.1379 & 0.6489 \\
\hline
\end{tabular}

Figure 2a shows the composite orthogonal pulse without noise interference. The analysis of the pulse in the presence of noise is also very important as noise interference may significantly vary the correlation values. Figure $2 b, c$ shows the composite pulses in the presence of noise at $10 \mathrm{~dB}$ and $20 \mathrm{~dB}$, respectively. Typical acceptable signal-to-noise ratio (SNR) values in wireless communication are around $20 \mathrm{~dB}$. SNR values greater than $40 \mathrm{~dB}$ are excellent, while $25 \mathrm{~dB}$ to $40 \mathrm{~dB}$ offers a very good signal, $15 \mathrm{~dB}$ to $25 \mathrm{~dB}$ is a low signal, $10 \mathrm{~dB}$ to $15 \mathrm{~dB}$ is a very low signal and $5 \mathrm{~dB}$ to $10 \mathrm{~dB}$ is equivalent to no signal at all.

Tables 6 and 7 present the correlation insight in the presence of noise (SNR $=10 \mathrm{~dB})$. Analyzing the correlation values in Tables 6 and 7 (SNR: $10 \mathrm{~dB}$ ), it is observed that the set threshold value of $21 \%$ will still hold true. The threshold value of $21 \%$ can accurately decode the presence of an orthogonal pulse in the given composite pulse in the presence of noise.

Similarly, Tables 8 and 9 show the correlation insight at the recommended SNR of $20 \mathrm{~dB}$. The correlation coefficient values in Tables 8 and 9 (with noise of $20 \mathrm{~dB}$ ) and Tables 4 and 5 (without noise) are very similar in terms of correlation values. 


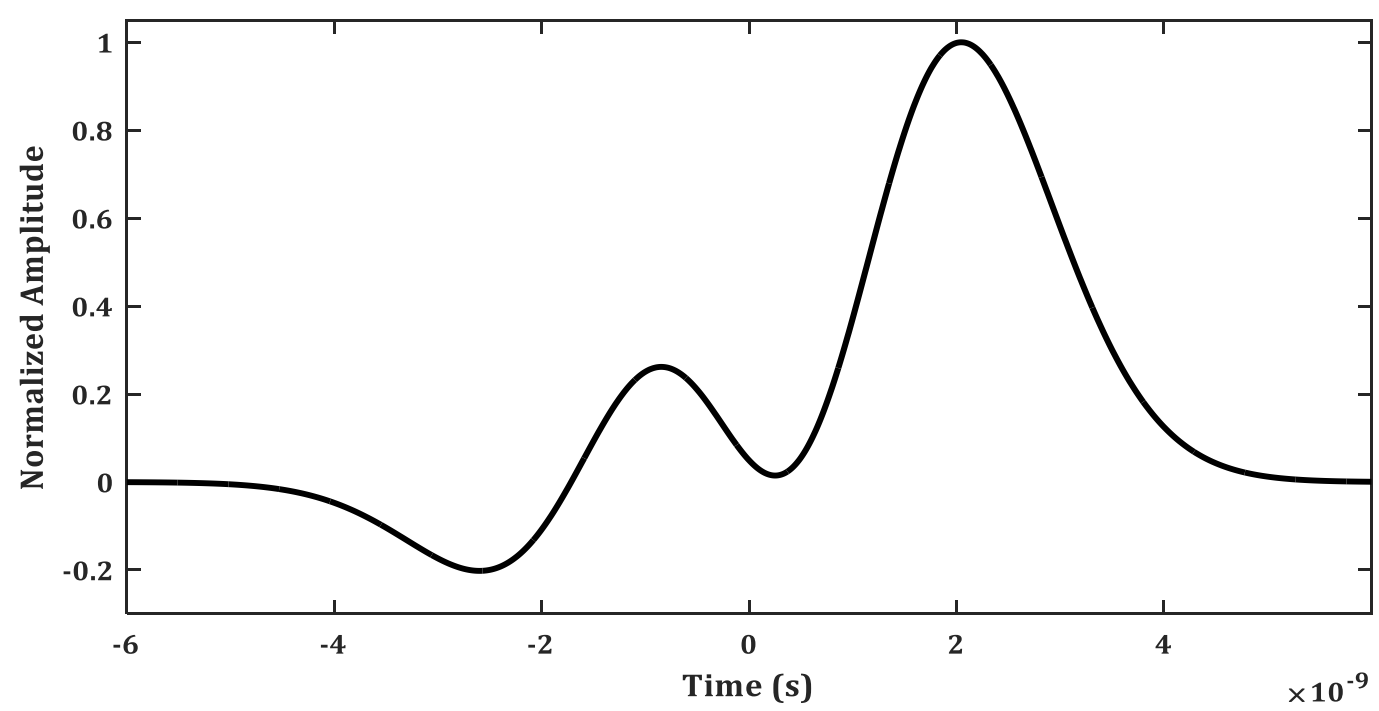

(a)

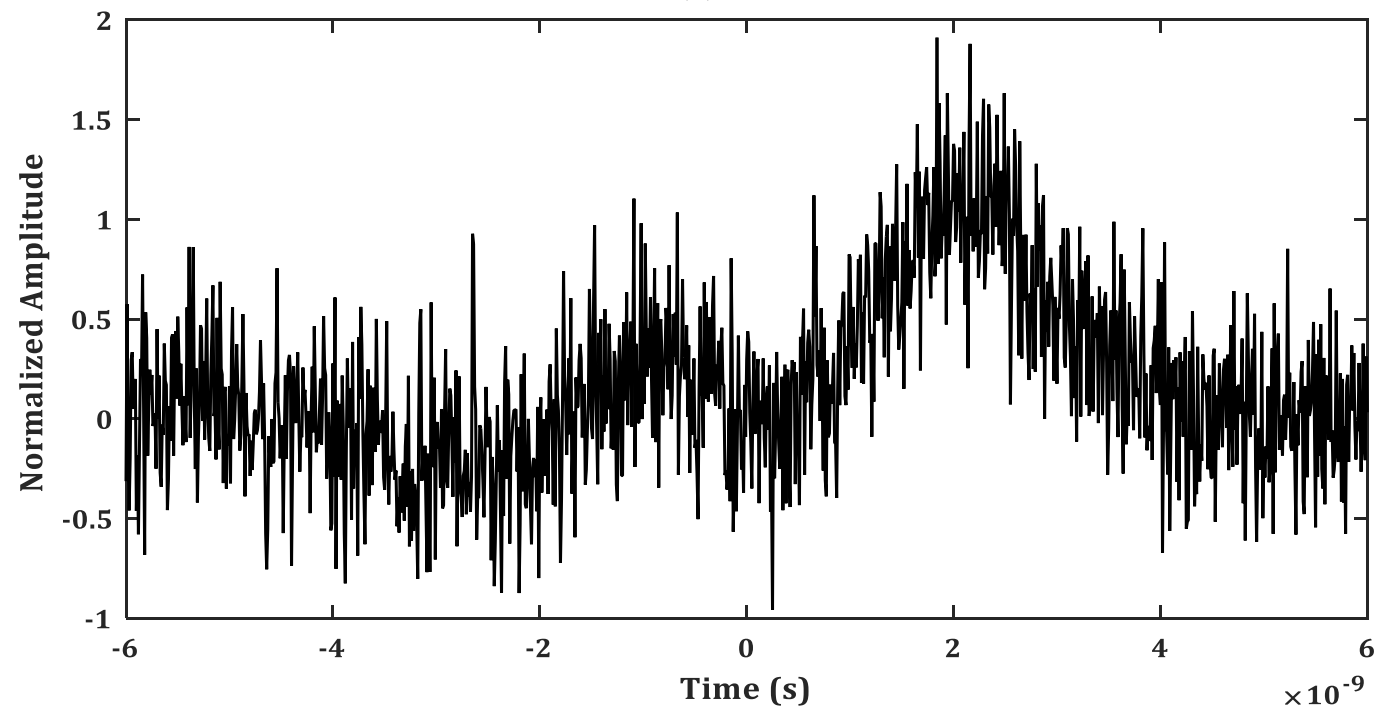

(b)

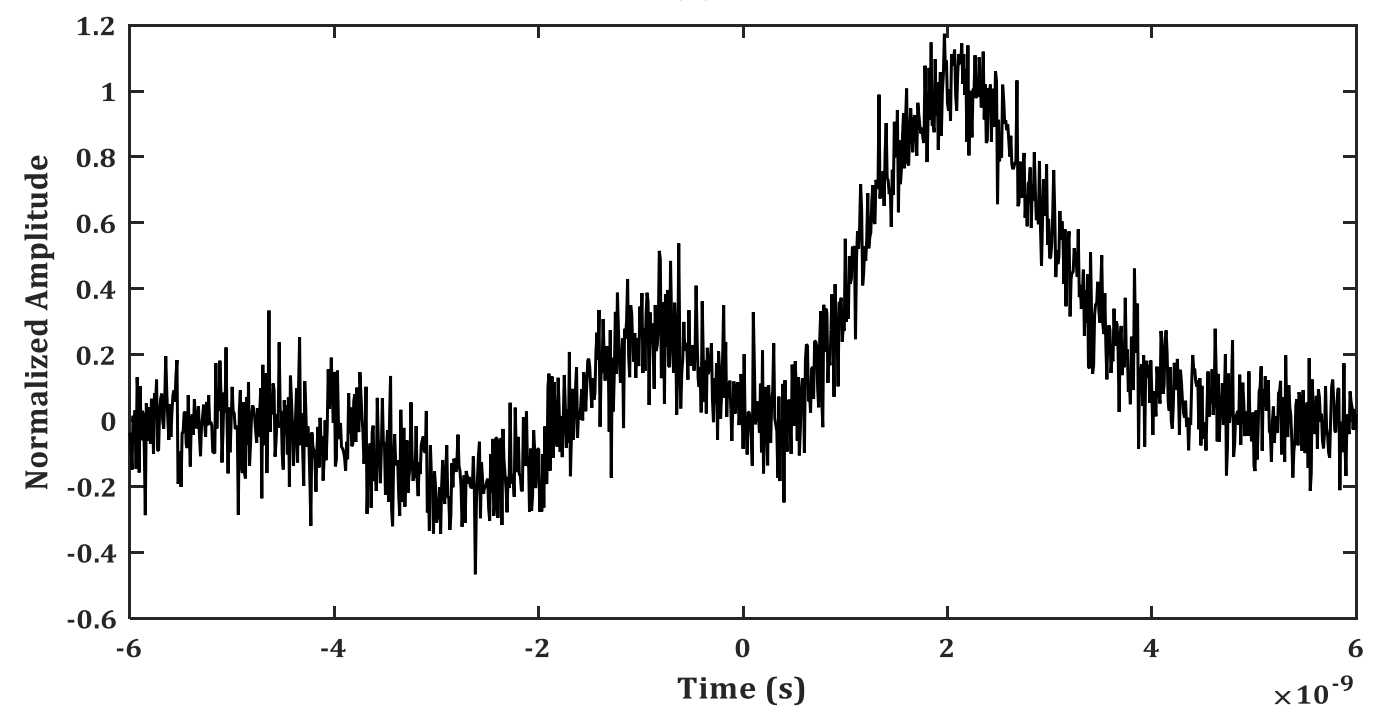

(c)

Figure 2. Composite pulse (a) without noise (b) with signal-to-noise ratio (SNR) $10 \mathrm{~dB}$ (c) with SNR $20 \mathrm{~dB}$. 
Table 6. Correlation coefficient values for selected pulses and all possible combinations of 2 orthogonal pulses with SNR of $10 \mathrm{~dB}$.

\begin{tabular}{ccccccc}
\hline & $\boldsymbol{M H P}_{\mathbf{0 , 1}}$ & $\boldsymbol{M H P}_{\mathbf{0 , 2}}$ & $\boldsymbol{M H P}_{\mathbf{0 , 3}}$ & $\boldsymbol{M H P}_{\mathbf{1 , 2}}$ & $\boldsymbol{M H P}_{\mathbf{1 , 3}}$ & $\boldsymbol{M H P}_{\mathbf{2 , 3}}$ \\
\hline$M H P_{0}$ & 0.5216 & 0.3963 & 0.5293 & -0.1727 & -0.0078 & -0.1640 \\
$M H P_{1}$ & 0.7807 & 0.0060 & 0.1487 & 0.6977 & 0.7470 & 0.1334 \\
$\mathrm{MH}_{2}$ & -0.1505 & 0.6911 & -0.1363 & 0.6459 & 0.0096 & 0.6360 \\
$M H P_{3}$ & 0.1383 & -0.0090 & 0.7858 & 0.1199 & 0.7444 & 0.7005 \\
\hline
\end{tabular}

Table 7. Correlation coefficient values for selected pulses and all possible combinations of 3 and 4 orthogonal pulses with SNR of $10 \mathrm{~dB}$.

\begin{tabular}{cccccc}
\hline & $\boldsymbol{M H P}_{\mathbf{1 , 2 , 3}}$ & $\boldsymbol{M H P}_{\mathbf{0 , 2 , 3}}$ & $\boldsymbol{M H P}_{\mathbf{0 , 1 , 3}}$ & $\boldsymbol{M H \boldsymbol { P } _ { \mathbf { 0 } , \mathbf { 1 } , \mathbf { 2 } }}$ & $\boldsymbol{M H P}_{\mathbf{0 , 1 , 2 , 3}}$ \\
\hline$M H P_{0}$ & -0.1354 & 0.3051 & 0.3922 & 0.2949 & 0.2163 \\
$M H P_{1}$ & 0.6213 & 0.1274 & 0.6753 & 0.6636 & 0.6065 \\
$M H P_{2}$ & 0.4886 & 0.5122 & -0.0954 & 0.5103 & 0.4019 \\
$M H P_{3}$ & 0.6227 & 0.1330 & 0.6812 & 0.1330 & 0.6227 \\
\hline
\end{tabular}

Table 8. Correlation coefficient values for selected pulses and all possible combinations of 2 orthogonal pulses with SNR of $20 \mathrm{~dB}$.

\begin{tabular}{ccccccc}
\hline & $\boldsymbol{M H P}_{\mathbf{0 , 1}}$ & $\boldsymbol{M H P}_{\mathbf{0 , 2}}$ & $\boldsymbol{M H P}_{\mathbf{0 , 3}}$ & $\boldsymbol{M H P}_{\mathbf{1 , 2}}$ & $\boldsymbol{M H P}_{\mathbf{1 , 3}}$ & $\boldsymbol{M H P}_{\mathbf{2 , 3}}$ \\
\hline$M H P_{0}$ & 0.5682 & 0.4409 & 0.5604 & -0.1725 & -0.0027 & -0.1739 \\
$M H P_{1}$ & 0.8154 & 0.0012 & 0.1660 & 0.7224 & 0.7657 & 0.1381 \\
$M H P_{2}$ & -0.1414 & 0.7424 & -0.1430 & 0.6794 & 0.0055 & 0.6759 \\
$M H P_{3}$ & 0.1563 & 0.0035 & 0.8165 & 0.1404 & 0.7710 & 0.7229 \\
\hline
\end{tabular}

Table 9. Correlation coefficient values for selected pulses and all possible combinations of 3 and 4 orthogonal pulses with SNR of $20 \mathrm{~dB}$.

\begin{tabular}{|c|c|c|c|c|c|}
\hline & $M H P_{1,2,3}$ & $M H P_{0,2,3}$ & $M H P_{0,1,3}$ & $M H P_{0,1,2}$ & $M H P_{0,1,2,3}$ \\
\hline$M H P_{0}$ & -0.1281 & 0.3209 & 0.3993 & 0.3089 & 0.2378 \\
\hline$M H P_{1}$ & 0.6510 & 0.1366 & 0.7042 & 0.6977 & 0.6356 \\
\hline $\mathrm{MHP}_{2}$ & 0.5190 & 0.5291 & -0.1015 & 0.5303 & 0.4046 \\
\hline $\mathrm{MHP}_{3}$ & 0.6572 & 0.7000 & 0.7066 & 0.1407 & 0.6452 \\
\hline
\end{tabular}

Thus, the selected threshold level of $21 \%$ satisfies both the low and high ends of the SNR values. With these results, it can be concluded that higher order orthogonal pulses can be generated using the modified Hermite pulse set generator. These orthogonal pulses can be added in time and then be transmitted as a composite pulse. It have been also shown that the composition of the composite pulse can be accurately determined with the threshold value of $21 \%$, even at the lower end SNR of $10 \mathrm{~dB}$.

\section{Single User UWB System}

With 4 orthogonal pulses, this scheme can represent 4 bits of data using 16 unique composite pulses. Each composite pulse can represent 4 bits of information. Table 10 shows the 4 bits of data and the corresponding composite pulses that can be used to represent them in a single time slot. In the receiving section, a correlator block can decode the composition of the received composite pulses and determine the transmitted 4 bits of data by analyzing the composition of the received pulses. This scheme will increase the data rate by 4 folds compared to the simple on-off keying (OOK)-based UWB communication system as a $300 \%$ gain is achieved. 
Table 10. Proposed scheme for data transmission using 4 orthogonal pulses in a single-user scenario.

\begin{tabular}{ccc}
\hline S.N. & Data & Pulse Representation \\
\hline 1. & 0000 & No pulse \\
2. & 0001 & $M H P_{0}$ \\
3. & 0010 & $M H P_{1}$ \\
4. & 0011 & $M H P_{2}$ \\
5. & 0100 & $M H P_{3}$ \\
6. & 0101 & $M H P_{0,1}$ \\
7. & 0110 & $M H P_{0,2}$ \\
8. & 0111 & $M H P_{0,3}$ \\
9. & 1000 & $M H P_{1,2}$ \\
10. & 1001 & $M H P_{1,3}$ \\
11. & 1010 & $M H P_{2,3}$ \\
12. & 1011 & $M H P_{0,1,2}$ \\
13. & 1100 & $M H P_{0,1,3}$ \\
14. & 1101 & $M H P_{0,2,3}$ \\
15. & 1110 & $M H P_{1,2,3}$ \\
16. & 1111 & $M H P_{0,1,2,3}$ \\
\hline
\end{tabular}

\section{Multiple-User UWB System}

For multiple channels, a unique pattern was assigned in order to encode data from each channel. In the proposed work, UWB MHP based on a pulse-encoding scheme is shown for simultaneous communication and higher data density. In the pulse-encoding scheme, instead of using conventional a single Gaussian pulse, a series of analog orthogonal pulses are utilized for simultaneous multi-channel support, and interference-free communication. For n-number of MHP orthogonal pulses, the channel number has been increased to $n(n-1)$. Hence, a unique pattern sequence has been developed by encoding the MHP orthogonal pulses to transmit binary high bits for each channel. By using 4 orthogonal pulses the proposed scheme can generate distinctive pattern sequences for 12 channels. The proposed scheme is different than the IFFT-based Walsh-Hadamard orthogonal code generation [60-69] where for a Hadamard matrix size of $\mathrm{n}$ results in $2^{n}$ number of channels. The main novelty of the proposed scheme is the reduced computational burden to generate the pulse-sequence as compared to the IFFT-based transform to generate the orthogonal vectors.

With 4 orthogonal pulses, the pattern will be a sequence of 4 unique orthogonal pulses. The receiver will be able to determine if a channel is transmitting binary 1 or 0 by matching the pattern, as each high bit (binary 1) from the channels will be represented by a unique sequence of 4 orthogonal pulses. The number of unique patterns that can be generated for a given number of orthogonal pulses is directed by,

$$
N=n(n-1)
$$

where $n$ is the number of orthogonal signals. For $n$ numbers of orthogonal pulse, each channel will have $n$ signal in the pattern. Tables 11-13 presents the different combinations of unique patterns for different number of orthogonal pulses.

As governed by the Equation (3), the number of unique patterns for 2, 3, and 4 orthogonal pulses is 2, 6, and 12 respectively. With 4 orthogonal pulses, 12 channels can be generated with 4-bit unique patterns. Each high bit (binary 1) from the channels will be represented by 4-bit sequence pattern as shown in Table 13. Each high bit from the channels will be spread into 4-bit unique sequences. This spread bits from all the channels will be added in time, resulting in a total of only 4 composite pulses from all the channels. The added 4 composite pulses will be transmitted in sequence. The transmitter architecture with 4 orthogonal pulses is shown in Figure 3. 
Table 11. Template pattern for 2 channels using 2 orthogonal pulses.

\begin{tabular}{cccc}
\hline Channel & First Bit & Second Bit & Pattern Sequence \\
\hline 1 & $M H P_{0}$ & $M H P_{1}$ & $M H P: 0,1$ \\
2 & $M H P_{1}$ & $M H P_{0}$ & $M H P: 1,0$ \\
\hline
\end{tabular}

Table 12. Template pattern for 6 channels using 3 orthogonal pulses.

\begin{tabular}{ccccc}
\hline Channel & First Bit & Second Bit & Third Bit & Pattern Sequence \\
\hline 1 & $M H P_{0}$ & $M H P_{1}$ & $M H P_{2}$ & $M H P: 0,1,2$ \\
2 & $M H P_{0}$ & $M H P_{1}$ & $M H P_{1}$ & $M H P: 0,1,1$ \\
3 & $M H P_{0}$ & $M H P_{2}$ & $M H P_{0}$ & $M H P: 0,0$ \\
4 & $M H P_{0}$ & $M H P_{0}$ & $M H P_{0}$ & $M H P: 0,0,0$ \\
5 & $M H P_{1}$ & $M H P_{1}$ & $M H P_{0}$ & $M H P: 1,1,0$ \\
6 & $M H P_{2}$ & $M H P_{1}$ & $M H P_{0}$ & $M H P: 2,1,0$ \\
\hline
\end{tabular}

Table 13. Template pattern for 12 channels using 4 orthogonal pulses.

\begin{tabular}{cccccc}
\hline Channel & First Bit & Second Bit & Third Bit & Fourth Bit & Pattern Sequence \\
\hline 1 & $M H P_{0}$ & $M H P_{1}$ & $M H P_{2}$ & $M H P_{3}$ & $M H P: 0,1,2,3$ \\
2 & $M H P_{0}$ & $M H P_{0}$ & $M H P_{1}$ & $M H P_{3}$ & $M H P: 0,0,1,3$ \\
3 & $M H P_{0}$ & $M H P_{2}$ & $M H P_{2}$ & $M H P_{3}$ & $M H P: 0,2,2,3$ \\
4 & $M H P_{0}$ & $M H P_{3}$ & $M H P_{2}$ & $M H P_{3}$ & $M H P: 0,3,2,3$ \\
5 & $M H P_{0}$ & $M H P_{0}$ & $M H P_{0}$ & $M H P_{3}$ & $M H P: 0,0,0,3$ \\
6 & $M H P_{0}$ & $M H P_{0}$ & $M H P_{2}$ & $M H P_{0}$ & $M H P: 0,0,2,0$ \\
7 & $M H P_{0}$ & $M H P_{0}$ & $M H P_{2}$ & $M H P_{1}$ & $M H P: 0,0,2,1$ \\
8 & $M H P_{0}$ & $M H P_{0}$ & $M H P_{2}$ & $M H P_{2}$ & $M H P: 0,0,2,2$ \\
9 & $M H P_{0}$ & $M H P_{0}$ & $M H P_{3}$ & $M H P_{3}$ & $M H P: 0,0,3$ \\
10 & $M H P_{1}$ & $M H P_{0}$ & $M H P_{2}$ & $M H P_{3}$ & $M H P: 1,0,2,3$ \\
11 & $M H P_{2}$ & $M H P_{0}$ & $M H P_{2}$ & $M H P_{3}$ & $M H P: 2,0,2,3$ \\
12 & $M H P_{3}$ & $M H P_{0}$ & $M H P_{2}$ & $M H P_{3}$ & $M H P: 3,0,2,3$ \\
\hline
\end{tabular}

The Figure 3 shows the logic of how the core section of the transmitter works for the proposed modulation scheme with 4 orthogonal pulses. Each bit from all the channels will be represented by the unique sequence of 4 orthogonal pulses. For instance, binary data ' 1 ' from channel 1 is represented by a sequence of MHP: 0, 1, 2, and 3. These individual bits from all the channels are then added in time in their respective positions by the composite symbol generator. These composite pulses contain the sum of different orthogonal pulses from all the channels, which are transmitted subsequently in the sequence illustrated in Figure 3. This will result in a total of 4 composite pulses for final transmission. With this modulation scheme, the data rate will be decreased as it takes 4 time slots to transmit 1 bit of information. However, at the cost of reduced data rate per channel, the number of particular channels used has been increased (12) to achieve simultaneous data transmission. This scheme employs 4 composite MHPs, each transmitting 12 bits of data in each time slot (e.g., symbol $1\left(S_{1}\right)$ ) which is a vast improvement over typical cases where it would take 12 time slots for transmitting individual bits of data from 12 channels. Hence, it can be said that there is a trade-off between the data rate and the number of channels. At the receiver end, there is a correlator block which is responsible for decoding the composite signals based on a set threshold value. It is followed by the logic block which matches the pattern and decides if binary 1 or 0 was transmitted from each channel. The receiver architecture with 4 orthogonal pulses is shown in Figure 4. The composite pulses are received in the order as they were transmitted. These composite pulses are sent to the correlator blocks to decode the composition of the pulses. After decoding, the decoded information is sent to the template matching block to decide if the particular channel has transmitted any information bits. Finally, the output from the template matching block is passed to the respective channels for further processing. There are 4 correlator blocks 
which are responsible for decoding the received pulses. Depending upon the power requirement and system complexity, the receiver architecture can be employed with either 4 correlator blocks or a single correlator block instead to decode the composite pulses in a sequential order. Doing so will enable the system to leverage the trade-off between speed and complexity of the system.

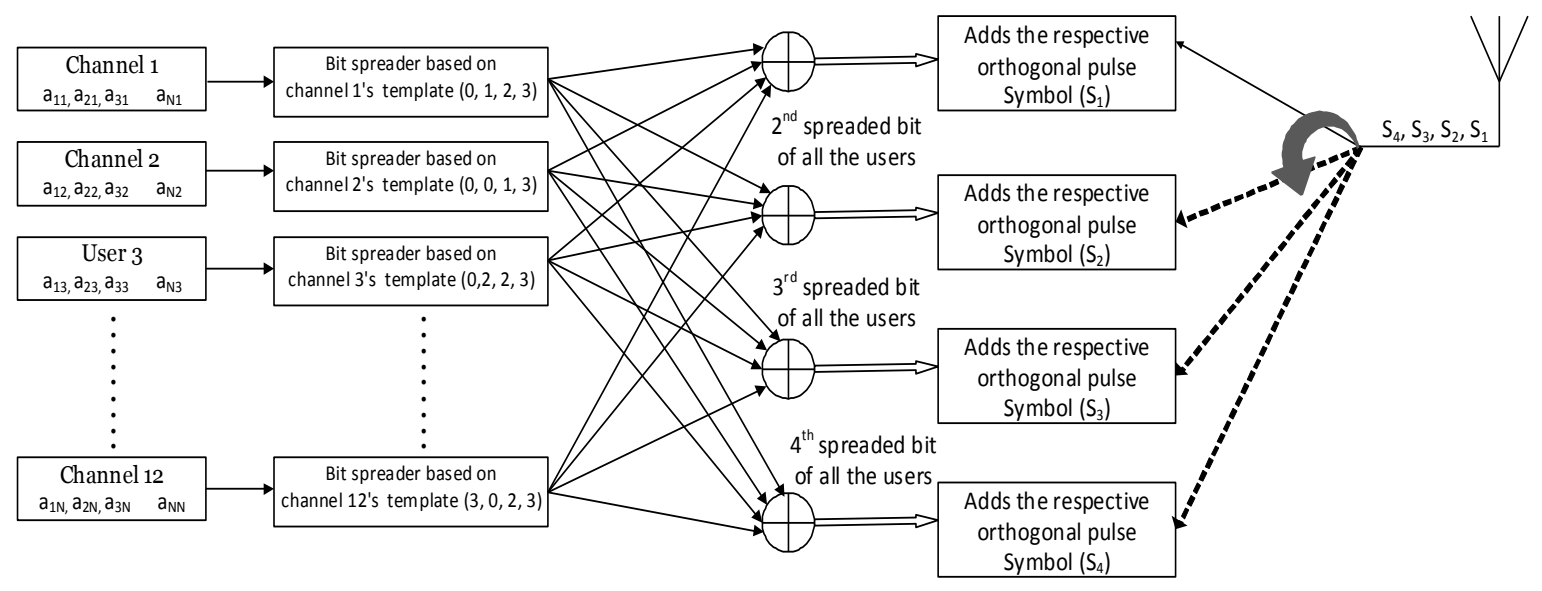

Figure 3. Proposed transmitter architecture for the ultra-wide band (UWB) system using pulse shape modulation (PSM).

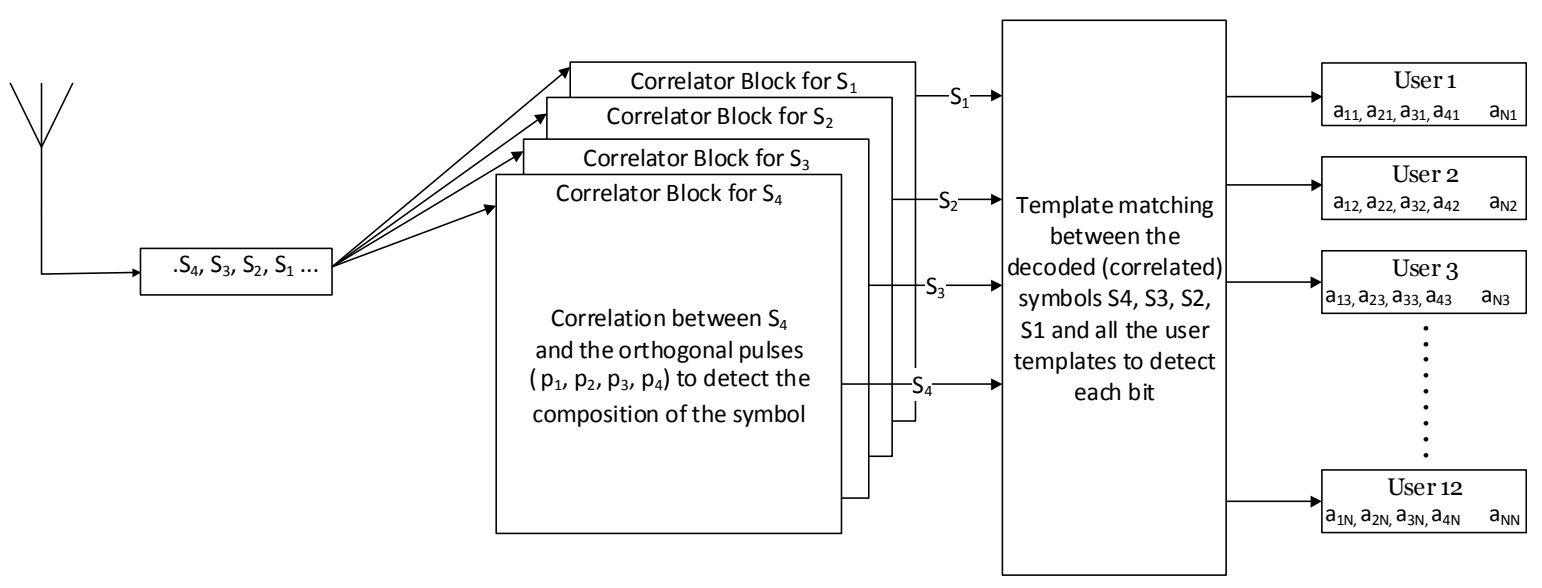

Figure 4. Proposed receiver architecture for the UWB system using PSM.

\section{BER Analysis}

Pulse shape modulation is more useful for M-ary communication where the individual orthogonal pulses are added together and transmitted as a composite pulse. The typical M-ary PSM symbol for the $k$ th user for the $i$ th symbol can be expressed as [56]:

$$
S_{i}^{(k)}(t)=\sqrt{E_{t x}^{(k)}} \sum_{j=0} W_{i}\left(t-j T_{f}-c_{j}^{(k)} T_{c}\right)
$$

where $W_{i}(t)$ is the orthogonal pulse, $T_{f}$ is the pulse repetition interval, $c_{j}^{(k)}$ is the pseudo random code for the $k$ th user in the $j$ th pulse repetition interval, $T_{c}$ is the chip duration, and $E_{t x}^{(k)}$ is the transmitted power of the $k$ th user. 
The multipath channel model proposed by the IEEE 802.15.3a can be expressed as the following discrete time impulse response [70]:

$$
h^{(k)}(t)=\sum_{l=1}^{L p} \alpha_{t}^{(k)} \delta\left(t-\tau_{l}^{k}\right)
$$

where, $\tau_{l}^{k}$ is the delay of the $k$ th user, and $\alpha_{t}^{(k)}$ is the $l$ th path gain of the $k$ th user assuming there are $L p$ number of total paths for all the users. If there are $N u$ number of users and each user experiences a different channel model, then the received signal can be expressed as :

$$
r(t)=\sum_{k=1}^{N u} \sum_{l=1}^{L p} \alpha_{l}^{(k)} S^{(k)}\left(t-\tau_{l}^{k}\right)+n(t)
$$

where $\tau_{l}^{k}$ is the time delay of the $l$ th path for the $k$ th user, $\alpha_{l}^{(k)}$ is the $l$ th path gain of the $k$ th user assuming there are $L p$ number of total paths for all the users, and $n(t)$ is the AWGN. Besides the AWGN, there could be sources of error from inter-symbol interference (ISI) due to inter-pulse interference (IPI), multiple-pulse interference (MPI), and multiple-access interference (MAI). The probability of error in the lth correlator in the presence of ISI and MAI can be expressed as:

$$
P_{l}=Q\left(\sqrt{\frac{\left(S_{q}\right)^{2}}{2\left(\sigma_{I P I}^{2}+\sigma_{M P I}^{2}+\sigma_{M A I}^{2}+\sigma_{N}^{2}\right)}}\right)
$$

where $Q$ is the error function, $S_{q}$ is the signal with $q$ th order pulse, $\sigma_{I P I}^{2}$ is the variance of IPI, $\sigma_{M P I}^{2}$ is the variance of MPI, $\sigma_{M A I}^{2}$ is the variance of MAI, and $\sigma_{N}^{2}$ is the variance of white noise component.

Each decision is independent; the average probability of bit error can be evaluated as:

$$
P_{\text {avg }}=\frac{1}{N} \sum_{l=0}^{N-1} P_{l}
$$

where $N$ is the total number of correlators in the $N$ bit symbol transmission. The correct decision of the $l$ th correlator is $\left(1-P_{l}\right)$. The decisions are independent, so the decision for a symbol can be defined as:

$$
P_{\text {avg }}=\prod_{l=0}^{N-1}\left(1-P_{l}\right)
$$

where, $\left(1-P_{c}\right)$ gives the error probability of the $N$ bit symbol.

Bit error rate (BER) is an important parameter while analyzing the performance of any communication system. It is a dimensionless quantity and is the ratio of bits with errors to the total number of bits transmitted over a given time. For the BER performance analysis, the complete system was developed in MATLAB and the analysis was carried out. Random message bits $\left(10^{6}\right)$ were generated and the number of erroneous bits at the receiver end was counted. The received signals at the receiver end were demodulated using the correlator block. The threshold value for the correlation was set to $21 \%$. This threshold value was chosen from the analysis of the correlation between the composite and the individual pulses. The BER plot obtained while transmitting random information bits transmission $\left(10^{6}\right)$ is shown in Figure 5.

From the BER plots it can be clearly observed that the bit error rate at the receiver end is $10^{-4}$ when the $E b / N_{0}$ is $13.8 \mathrm{~dB}$. The reason for the bit being erroneous bit is that the threshold is hard set to 0.21 . However, if the threshold value can be set to be adaptive depending upon the channel condition, the BER performance will be significantly improved. 


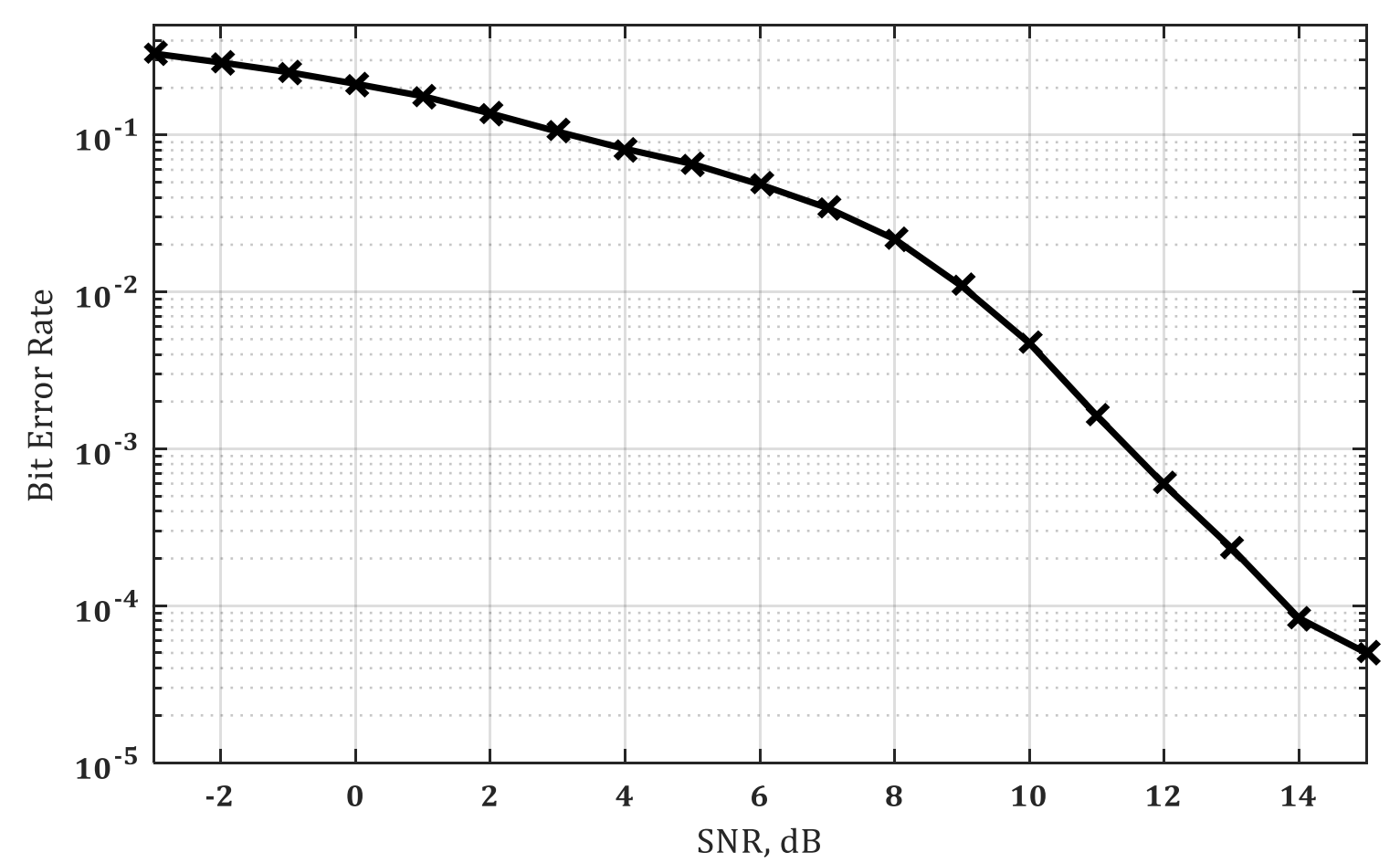

Figure 5. Bit error rate (BER) plot for pulse shape modulation for random inputs $\left(10^{6}\right)$.

\section{Conclusions}

Two novel schemes for data rate enhancement in UWB communication technology have been presented through the course of this paper. The proposed schemes use orthogonal pulses to transmit data in both single user and multiple user scenarios. Instead of transmitting individual orthogonal pulses, this scheme transmits composite pulse of 4 orthogonal pulses and by doing such it manages to increase the data rate in single-user scenario by 4 folds and at the same time accommodates 12 channels for simultaneous data transmission. The correlation properties of the MHP are analyzed through MATLAB simulations as it also validates that the composite pulse can be decoded in the receiver end with a threshold value calculated from the correlation analysis of MHPs with a 10-ns pulse width. The carefully chosen threshold level of $21 \%$ satisfies both the low and high ends of the SNR values. One of the major gains in employing the proposed scheme is its capability to work with the existing pulse-based UWB system due to the similarities in features such as the pulse width, pulse repetition interval (PRI), and energy with that of the single orthogonal pulse-based system. Since the difference exists exclusively in the shape of the pulse, it will not affect the transmission and the synchronization; as a result the proposed scheme can be readily incorporated with the existing UWB system with minimal change. The proposed scheme's competence in enhancing number of channels for simultaneous data transmission along with the rate of data being transmitted in sub-GHz UWB communication makes it a leading alternative for future wireless sensing applications.

Acknowledgments: Partial support of this work was provided by the NSF-CPS (Cyber-Physical Systems) program with award number CNS-1645863. Opinions expressed are those of the authors and not necessarily those of the National Science Foundation.

Author Contributions: K.P.P., Y.-G.L. and M.R.H. contributed to the algorithm design and simulation; A.K.M.A. contributed to the simulation and writing of the paper.

Conflicts of Interest: The authors declare no conflict of interest. 


\section{References}

1. Chiani, M.; Giorgetti, A. Coexistence between UWB and narrow-band wireless communication systems. Proc. IEEE 2009, 97, 231-254.

2. Giorgetti, A.; Chiani, M.; Win, M.Z. The effect of narrowband interference on wideband wireless communication systems. IEEE Trans. Commun. 2005, 53, 2139-2149.

3. Pinto, P.C.; Giorgetti, A.; Win, M.Z.; Chiani, M. A stochastic geometry approach to coexistence in heterogeneous wireless networks. IEEE J. Sel. Areas Commun. 2009, 27, 1268-1282.

4. Giorgetti, A.; Sithamparanathan, K. Cognitive Radio Techniques: Spectrum Sensing, Interference Mitigation, and Localization; Artech House: Norwood, MA, USA, 2012.

5. Giorgetti, A. Interference Mitigation Technique by Sequence Design in UWB Cognitive Radio. In Proceedings of the 2010 3rd International Symposium on Applied Sciences in Biomedical and Communication Technologies (ISABEL), Rome, Italy, 7-10 November 2010; pp. 101-105.

6. Zhang, H.; Li, W.; Gulliver, T.A. Pulse Position Amplitude Modulation for Time-Hopping Multiple-Access UWB Communicatios. IEEE Trans. Commun. 2005, 53, 1269-1273.

7. Zhang, H.; Gulliver, T.A. Biorthogonal Pulse Position Modulation for Time-Hopping Multiple-Access UWB Communicatios. IEEE Trans. Wirel. Commun. 2005, 4, 1154-1162.

8. Majhi, S.; Madhukumar, A.S.; Premkumar, A.B.; Richardson, P. Combining OOK with PSM Modulation for TH-UWB Radio Systems: A Performance Analysis. EURASIP J. Wirel. Commun. Netw. 2008, 1-11, doi:10.1155/2008/735410.

9. Revision of Part 15 of the Commission's Rules Regarding Ultra-wideband Transsmision Systems; Federal Communications Commission: Washington, DC, USA, 2002; pp. 98-153.

10. Kim, Y.; Jang, B.; Shin, C.; Womack, B.F. Orthonormal Pulses for High Data Rate Communications in Indoor UWB Systems. IEEE Commun. Lett. 2005, 9, 405-407.

11. Da Silva, J.A.N.; de Campos, M.L.R. Method for Obtaining Spectrally Efficient Orthogonal UWB Pulse Shapes. In Proceedings of the International Telecommunications Symposium (ITS2006), Fortaleza-CE, Brazil, 3-6 September 2006; pp. 812-817.

12. Li, Y.G.; Haider, M.R.; Massoud, Y. An efficient orthogonal pulse set generator for high-speed sub-GHz UWB communications. In Proceedings of the IEEE International Symposium on Circuits and Systems, Melbourne, Australia, 1-5 June 2014; pp. 1913-1916.

13. Usuda, K.; Honggang, Z.; Nakagawa, M. M-ary pulse shape modulation for PSWF-based UWB systems in multipath fading environment. In Proceedings of the IEEE Global Telecommunications Conference, Dallas, TX, USA, 29 November-3 December 2004; pp. 3498-3504.

14. Youngok, K.; Byungchul, J.; Changyong, S.; Womack, B.F. Orthonormal pulses for high data rate communications in indoor UWB systems. IEEE Commun. Lett. 2005, 9, 405-407.

15. Win, M.Z.; Scholtz, R.A. Ultra-Wide Bandwidth time-hopping spread-spectrum impulse radio for wireless multiple-access communications. IEEE Trans. Commun. 2000, 48, 679-691.

16. Wu, X.; Tian, Z.; Davidson, T.N.; Giannakis, G.B. Optimal waveform design for UWB radios. In Proceedings of the IEEE International Conference on Acoustics, Speech, and Signal Processing, Montreal, QC, Canada, 17-21 May 2004; pp. 521-524.

17. Luo, X.; Yang, L.; Giannakis, G.B. Designing optimal pulse-shapers for ultrawideband radios. In Proceedings of the 2003 IEEE Conference on Ultra Wideband Systems and Technologies, Reston, VA, USA, 16-19 November 2003; pp. 349-353.

18. Sheng, H.; Orlik, P.; Haimovich, A.M.; Cimini, L.J., Jr.; Zhang, J. On the spectral and power requirements for ultra-wideband transmission. In Proceedings of the IEEE International Conference on Communications, Anchorage, AK, USA, 11-15 May 2003; pp. 738-742.

19. De Nardis, L.; Giancola, G.; Benedetto, M.G.D. Power limits fulfilment and MUI reduction based on pulse shaping in UWB networks. In Proceedings of the 2004 IEEE International Conference on Communications, Paris, France, 20-24 June 2004; pp. 3576-3580.

20. Beaulieu, N.C.; Hu, B. A novel pulse design algorithm for ultrawideband communications. In Proceedings of the Global Telecommunications Conference, Dallas, TX, USA, 29 November-3 December 2004; pp. 3220-3224.

21. Parr, B.; Cho, B.; Wallace, K.; Ding, Z. A novel ultra-wideband pulse design algorithm. IEEE Commun. Lett. 2003, 7, 219-221. 
22. Ghavami, M.; Michael, L.; Kohno, R. Ultra Wideband Signals and Systems in Communication Engineering, 2nd ed.; John Wiley \& Sons: Hoboken, NJ, USA, 2007.

23. Ghavami, M.; Michael, L.B.; Haruyama, S.; Kohno, R. A novel UWB pulse shape modulation system. Wirel. Pers. Commun. 2002, 23, 105-120.

24. Michael, L.; Ghavami, M.; Kohno, R. Multiple pulse generator for ultra-wideband communication using hermite polynomial based orthogonal pulses. In Proceedings of the IEEE Conference on Ultra Wideband Systems and Technologies, Baltimore, MD, USA, 21-23 May 2002; pp. 47-51.

25. Gao, Y.; Zheng, Y.; Diao, S.; Toh, W.; Ang, C.; Je, M.; Heng, C. Low-Power Ultrawideband Wireless Telemetry Transceiver for Medical Sensor Applications. IEEE Trans. Biomed. Eng. 2011, 58, 768-772.

26. Chae, M.S.; Yang, Z.; Yuce, R.M.; Hoang, L.; Liu, W. A 128-Channel 6 mW Wireless Neural Recording IC With Spike Feature Extraction and UWB Transmitter. IEEE Trans. Neural Syst. Rehabil. Eng. 2009, 17, 312-321.

27. Haider, M.R.; Islam, A.B.; Islam, S.K. Ultra-lower-power sensor signal monitoring and impulse radio architecture for biomedical applications. In Proceedings of the IEEE Annual Symposium on VLSI, (ISVLSI2010), Lixouri Kefalonia, Greece, 5-7 July 2010; pp. 222-227.

28. Tandall, T.; Mahbub, I.; Islam, A.B.; Haider, M.R.; Islam, S.K. Low-power sensor signal monitoring and impulse radio architecture for biomedical applications. In Analog Integrated Circuits and Signal Processing; Springer: Berlin/Heidelberg, Germany, 2014; Volume 78, pp. 209-216.

29. Greenwald, E.; Mohsen, M.; Nitish, T.; Tang, W.; Culurciello, E. A VLSI neural monitoring system with ultra-wideband telemetry for awake behaving subjects. In Proceedings of the 2010 IEEE International Symposium on Circuits and Systems (ISCAS), Paris, France, 30 May-2 June 2010; pp. 1193-1196,.

30. Soldá, S.; Caruso, M.; Bevilacqua, A.; Gerosa, A.; Vogrig, D.; Neviani, A. A 5 Mb/s UWB-IR Transceiver Front-End for Wireless Sensor Networks in $0.13 \mu \mathrm{m}$ CMOS. IEEE J. Solid-State Circuits 2011, 46, 1636-1647.

31. Crepaldi, M.; Li, C.; Dronso, K.; Fernandes, J.; Kinget, P. An ultralow-power interference-robust IR-UWB transceiver chipset using self-synchronizing OOK modulation. In Proceedings of the Solid-State Circuits Conference Digest of Technical Papers (ISSCC), San Francisco, CA, USA, 7-11 February 2010; pp. 226-228.

32. Daly, D.C.; Mercier, P.P.; Bhardwaj, M.; Stone, A.L.; Aldworth, Z.N.; Daniel, T.L.; Voldman, J.; Hildebrand, J.G.; Chandrakasan, A.P. A Pulsed UWB Receiver SoC for Insect Motion Control. IEEE J. Solid-State Circuits 2010, 45, 153-166.

33. PMercier, P.; Daly, D.C.; Chandrakasan, A.P. An energy-efficient all-digital UWB transmitter employing dual capacitively-coupled pulse-shaping drivers. IEEE J. Solid-State Circuits 2009, 44, 1679-1688.

34. Park, Y.; Wentzloff, D. An all-digital 12 pJ/pulse IR-UWB transmitter synthesized from a standard cell library. IEEE J. Solid-State Circuits 2011, 46, 1147-1157.

35. Majidzadeh, V.; Schmid, A.; Leblebici, Y.; Rabaey, J. An 8-PPM, 45 pJ/bit UWB transmitter with reduced number of PA elements. In Proceedings of the 2012 Symposium on VLSI Circuits (VLSIC), Honolulu, HI, USA, 13-15 June 2012; pp. 36-37.

36. Lee, F.S.; Chandrakasan, A.P. A 2.5 nJ/bit 0.65 V Pulsed UWB Receiver in $90 \mathrm{~nm}$ CMOS. In Proceedings of the Digest of Technical Papers. IEEE International Solid-State Circuits Conference, San Francisco, CA, USA, 1-15 February 2007.

37. Lachartre, D.; Denis, B.; Morche, D.; Ouvry, L.; Manuel, P. B.; Piaget, B.; Prouvée, J.; Vincent, P. A 1.1nJ/bit 802.15.4a-Compliant Fully Integrated UWB Transceiver in 0.13- $\mu \mathrm{m}$ CMOS. In Proceedings of the IEEE International Solid-State Circuits Conference-Digest of Technical Papers, San Francisco, CA, USA, 8-12 February 2009.

38. Werther, O.; Cavin, M.; Schneider, A.; Renninger, R.; Liang, B.; Bu, L.; Jin, Y.; Rogers, J.; Marcincavage, J. A Fully Integrated 14 Band, 3.1 to $10.6 \mathrm{GHz} 0.13 \mu \mathrm{m}$ SiGe BiCMOS UWB RF Transceiver. IEEE J. Solid-State Circuits 2008, 43, 2829-2843.

39. Diao, S.; Zheng, Y.; Gao, Y.; Cheng, S.; Yuan, X.; Je, M.; Heng, C. A 50-Mb/s CMOS QPSK/O-QPSK Transmitter Employing Injection Locking for Direct Modulation. IEEE Trans. Microw. Theory Tech. 2012, 60, 120-130.

40. Joo, S.; Chen, W.-H.; Choi, T.-Y.; Oh, Mi.-K.; Park, J.-H.; Kim, J.-Y.; Jung, B. A fully integrated 802.15.4 a IR-UWB transceiver in $0.13 \mu \mathrm{m}$ CMOS with digital RRC synthesis. In Proceedings of the 2010 IEEE International Solid-State Circuits Conference Digest of Technical Papers (ISSCC), San Francisco, CA, USA, 7-11 February 2010; pp. 228-230. 
41. Fernandes, J.R.; Wentzloff, D. Recent Advances in IR-UWB Transceivers: An Overview. In Proceedings of the IEEE International Symposium on Circuits and Systems, Paris, France, 30 May-2 June 2010; pp. 3284-3287.

42. Arbabian, A.; Kang, S.; Callender, S.; Afshar, B.; Chien, J.-C.; Niknejad, A.M. A 90 GHz pulsed-transmitter with near-field/far-field energy cancellation using a dual- loop antenna. In Proceedings of the IEEE Radio Frequency Integrated Circuits Symposium (RFIC), Baltimore, MD, USA, 5-7 June 2011; pp. 1-4.

43. Nejad, M.B. Ultra Wideband Impulse Radio for Wireless Sensing and Identification. Ph.D. Thesis, KTH Royal Institute of Technology (KTH), Stockholm, Sweden, 2008.

44. Ryckaert, J.; Desset, C.; Fort, A.; Badaroglu, M.; de Heyn, V.; Wambacq, P.; van der Plas, G.; Donnay, S.; van Poucke, B.; Gyselinckx, B. Ultra-wideband transmitter for low-power wirless body area networks: Design and evaluation. IEEE Trans. Circuits Syst. I Regul. Pap. 2005, 52, 2515-2525.

45. Gao, Y.; Diao, S.; Ang, C.-W.; Zheng, Y.; Yuan, X. Low Power Ultra-wideband Wireless Telemetry System for Capsule Endoscopy Application. In Proceedings of the IEEE Conference on Robotics, Automation and Mechatronics, Singapore, 28-30 June 2010; pp. 96-99.

46. Dardari, D.; Conti, A.; Ferner, U.; Giorgetti, A.; Win, M.Z. Ranging with ultra-wide bandwidth signals in multipath environments. Proc. IEEE 2009, 97, 404-426.

47. Gerrits, J.F.M.; Farserotu, J.R.; Long, J.R. Low-Complexity Ultra-Wide-Band Communications. IEEE Trans. Circuits Syst. II Express Briefs 2008, 55, 329-333.

48. Crepaldi, M.; Ros, P.M.; Bonanno, A.; Morello, M.; Demarchi, D. A non-coherent IR-UWB receiver for high sensitivity short distance estimation. In Proceedings of the 2014 IEEE International Symposium on Circuits and Systems (ISCAS), Melbourne, Australia, 1-5 June 2014; pp. 1905-1908.

49. Arbabian, M.A. Time-Domain Ultra-Wideband Synthetic Imager in Silicon. Ph.D. Thesis, University of California at Berkeley, Berkeley, CA, USA, 2013.

50. Joo, Y.; Kim, H.; Jung, S. A tunable pulse generator for sub-ghz uwb systems. In Proceedings of the 52nd IEEE International Midwest Symposium on Circuits and Systems, Cancun, Mexico, 2-5 August 2009; pp. $292-296$.

51. Stoopman, M.; Serdijn, W.A. A sub-GHz UWB pulse generator for wireless implantable medical devices. In Proceedings of the IEEE Biomedical Circuits and Systems Conference, San Diego, CA, USA, 10-12 November 2011; pp. 149-152.

52. Zasowski, T.; Althaus, F.; Wittneben, A. Temporal cognitive UWB medium access in the presence of multiple strong signal interferers. In Proceedings of the 14th IST Mobile Wireless Commun, Dresden, Germany, 19-23 June 2005.

53. Yu, P.; Schaumont, P.; Ha, D. Securing RFID with Ultra-Wideband Modulation. In Proceedings of the RFID Sec 2006 Workshop on RFID Security, Graz, Austria, 12-14 July 2006.

54. Mancilla, D.; Moczygemba, J. Exploring Medical Identity Theft. Perspect. Health Inf. Manag. 2009, 6, 1-11.

55. Zhang, L.; Zhou, Z. Research on orthogonal wavelet synthesized uwb waveform signal. In Proceedings of the IEEE International Symposium on Communications and Information Technology, Beijing, China, 12-14 October 2005; Volume 2, pp. 830-832.

56. Majhi, S.; Madhukumar, A.S.; Premkumar, A.B.; Chin, F. Modulation Schemes Based on orthogonal pulse for time hopping Ultrawide band radio systems. In Proceedings of the IEEE Conference on Communications, Glasgow, UK, 24-28 June 2007; pp. 4185-4190.

57. Dilmaghani, R.; Ghavami, M.; Aghvami, A. Uwb multiple-pulse generator and transmitter. In Proceedings of the 2004 International Workshop on Ultra Wideband Systems, Joint with Conference on Ultrawideband Systems and Technologies, Kyoto, Japan, 18-21 May 2004; pp. 117-121.

58. Gharwal, A. Ultra Wideband Pulse Generation Using Discrete Prolate Spheroidal Sequences: Design and Analyses. Master's Thesis, San Diego State University, San Diego, CA, USA, 2012.

59. Allen, B.; Ghorashi, S.; Ghavarm, M. A review of pulse design for impulse radio. In IEE Seminar on Ultra Wideband Communications Technologies and System Design; The Institution of Engineering and Technology: Stevenage, UK, 2004; pp. 93-97.

60. Del Re, E.; Fantacci, R.; Ronga, L.S. Fast phase sequences spreading codes for CDMA using FFT. In Proceedings of the 9th European Signal Processing Conference (EUSIPCO 1998), Rhodes, Greece, 8-11 September 1998; pp. 1-5.

61. Molisch, A.F. Wireless Communications, 2nd ed.; John Wiley \& Sons: Chichester, UK, 2011; pp. 417-443.

62. Li, Y.H. Design of a Walsh Function Generator. IEEE Trans. Electromagn. Compat. 1987, EMC-29, 83-86, doi:10.1109/TEMC.1987.304340. 
63. Alsusa, E.; Yang, L. Selective post-IFFT amplitude randomising for peak-to-average power ratio reduction in orthogonal frequency-division multiplexing-based systems. IET Commun. 2008, 2, 553-561, doi:10.1049/iet-com:20070098.

64. Zou, D.; Lu, X.; Wu, H.; Xu, J. Application of IFFT Based on CORDIC Algorithms in OFDM-UWB System. In Proceedings of the 2008 4th International Conference on Wireless Communications, Networking and Mobile Computing, Dalian, China, 12-14 October 2008; pp. 1-5.

65. Ishebabi, H.; Ascheid, G.; Meyr, H.; Atak, O.; Atalar, A.; Arikan, E. An efficient parallelization technique for high throughput FFT-ASIPs. In Proceedings of the 2006 IEEE International Symposium on Circuits and Systems, Island of Kos, Greece, 21-24 May 2006; p. 4.

66. Li, B.; Zhou, Z.; Zou, W.; Li, D.; Zhao, C. Optimal Waveforms Design for Ultra-Wideband Impulse Radio Sensors. Sensors 2010, 10, 11038-11063; doi:10.3390/s101211038

67. Vahid, M.B. Low-Power Circuits and Systems Design for Data Acquisition and Transmission in a Wireless Cortical Implant. Ph.D. Thesis, École Polytechnique Fédérale de Lausanne (EPFL), Lausanne, Switzerland, 2012.

68. Majidzadeh, V.; Schmid, A.; Leblebici, Y. A 16-Channel, $359 \mu$ W, Parallel Neural Recording System Using Walsh-Hadamard Coding. In Proceedings of the IEEE 2013 Custom Integrated Circuits Conference, San Jose, CA, USA, 22-25 September 2013; pp. 1-4.

69. Mirahmadi, M.; Al-Dweik, A.; Shami, A. BER Reduction of OFDM Based Broadband Communication Systems over Multipath Channels with Impulsive Noise. IEEE Trans. Commun. 2013, 61, 4602-4615, doi:10.1109/TCOMM.2013.102313.130220.

70. Meystre, S. The Current State of Telemonitoring: A Comment on the Literature. Telemed. E-Health 2005, 11, 63-69.

(C) 2017 by the authors. Licensee MDPI, Basel, Switzerland. This article is an open access article distributed under the terms and conditions of the Creative Commons Attribution (CC BY) license (http://creativecommons.org/licenses/by/4.0/). 